\title{
Hurst's Rescaled Range Statistical Analysis for Pseudorandom Number Generators used in Physical Simulations
}

\author{
B. M. Gammel \\ Physik Department der Technischen Universität München, T30, 85747 Garching, Germany \\ August 7, 1997
}

\begin{abstract}
The rescaled range statistical analysis $(R S)$ is proposed as a new method to detect correlations in pseudorandom number generators used in Monte Carlo simulations. In an extensive test it is demonstrated that the RS analysis provides a very sensitive method to reveal hidden long run and short run correlations. Several widely used and also some recently proposed pseudorandom number generators are subjected to this test. In many generators correlations are detected and quantified.
\end{abstract}

PACS numbers: 02.70.Lq, 05.40.+j, 02.50.-r, 75.40.Mg

\section{INTRODUCTION}

Random numbers are the essential ingredient of all stochastic simulations. A great many algorithms in Monte-Carlo (MC) simulations and other non-physical computational fields rely crucially on the statistical properties of the random numbers used. High precision calculations on nowadays computer hardware typically involve the generation of billions of random numbers.

Today the most convenient and most reliable method of obtaining random numbers in practice is the use of a deterministic algorithm. Such a numerical method produces a sequence of pseudorandom numbers (PRN) which mimic the statistical properties of true random numbers as good as possible. Usually the pseudorandom number generator (PRNG) is assumed to generate a sequence of independent and identically distributed continuous $\mathrm{U}(0,1)$ random number, that means uniformly distributed over the interval $(0,1)$. Other distributions can be obtained by transformation methods [1]. Since the state space of the generator is finite the sequence of PRNs will be eventually periodic. Therefore the expected properties of "true" random variables can only be approximated.

True random numbers can only be produced by physical devices that generate events which are principally unpredictable in advance, such as noise diodes or gamma ray counters. But such devices are inconvenient to use and Marsaglia reported that several commercial products fail standard statistical tests spectacularly 22,3]. An alternative could be the archiving of random numbers of high quality on a CDROM [2], although such a source is by far not as convenient to handle as a simple function call.

While theoretical test methods [4.5], such as the analysis of the lattice structure [6] of linear congruential generators, are certainly the starting point for constructing a good PRNG there is also a strong need for so-called empirical tests. These view the PRNG under consideration as a black box and statistically analyze sequences of numbers for various types of correlations, regardless of the generation method. There is a large battery of stan- dard tests [3 5, 0,8,2] which every candidate to be used in "serious" simulations has to pass. PRNGs that have succeeded in all of these tests seemed to work reliable in apparently all physical simulations until the last few years. But the rapid development of computer hardware and improved simulation algorithms have caused the demands on the quality of the random number sequences to greatly increase. As a consequence erroneous results have been found in recent high precision MC calculations. The errors could be related to the use of popular PRNGs in combination with some specialized algorithms [9] 13] which revealed hitherto undetected correlations in the pseudorandom sequences.

Thus there is a strong need to enlarge the tool box of empirical tests to gain confidence in newly proposed PRNGs 14 17 and to check whether traditionally used PRNGs are still reliable in modern applications. Any good statistical test should have an idiosyncracy for unwanted correlations and detect defects before they show up in an application. Newly developed and highly specialized algorithms may be sensitive to structural defects in PRNGs which are not evident in the standard tests. As different tests detect different types of defects it is desirable to develop application specific tests 182 21] that are especially sensitive to the features of the random numbers which are probed in simulations in current fields of research. But often this cannot be assessed in advance and the only way to reassure oneself of the correctness of a suspicious (or very important) result is to perform an in situ test and to repeat the simulation with some different PRNGs. Enlarging the set of test methods therefore can help to save precious time and to avoid painful recalculations.

In section II a new test method is proposed which is applied to a set of several popular generators described in section III. In section IV the results of the numerical experiments are discussed illustrating the capability of the new test. Following the conclusions, section $\mathrm{V}$. additional results are tabulated in the appendices. 


\section{THE RS ANALYSIS}

In the following I describe a new technique for judging the quality of PRNGs in at least several physically relevant situations. It will be demonstrated that the rescaled range statistical analysis ( $R S$ analysis) provides an extremely sensitive method for revealing hidden correlations in PRNGs.

As this method is based on general statistical properties expected for an independent Gaussian process it should also be useful as a general tool to test the suitability of a PRNG in a wide class of stochastic simulations. In the sequel it will be shown that it is especially effective for testing the presence of long run statistical dependence and in cases where such a correlation is present, for estimating its intensity. In addition it is shown that also short run cyclic components in a pseudorandom sequence are easily made evident using the $R S$ statistic.

Hydrology is the oldest discipline in which noncyclic long run dependence has been reported. In particular the $R S$ analysis has been invented by Hurst [22,23] when he was studying the Nile in order to describe the long term dependence of the water level in rivers and reservoirs. Later his method has gained much attention in the context of fractional Brownian motion 24.

The $R S$ statistic for a series $\xi_{t}$ in the discrete integer valued time is conventionally defined as follows:

$$
\begin{aligned}
X(t, s) & =\sum_{u=1}^{t}\left(\xi_{u}-\langle\xi\rangle_{s}\right) \\
R(s) & =\max _{1 \leq t \leq s} X(t, s)-\min _{1 \leq t \leq s} X(t, s) \\
S(s) & =\left[\frac{1}{s} \sum_{t=1}^{s}\left(\xi_{t}-\langle\xi\rangle_{s}\right)^{2}\right]^{\frac{1}{2}} \\
R S(s) & =R(s) / S(s)
\end{aligned}
$$

Viewing the $\xi_{t}$ as spatial increments in a onedimensional random walk then $\sum_{t=1}^{s} \xi_{t}$ is the distance of the walker from the starting point at time $s$. In the quantity $X(t, s)$ the mean

$$
\langle\xi\rangle_{s}=\frac{1}{s} \sum_{t=1}^{s} \xi_{t}
$$

over the time lag $s-1$ is subtracted to remove a trend if the expectation value of $\xi_{t}$ is not zero. In the sequel the difference between the final time $s$ and the initial time 1 of the stochastic process will be termed the lag $\tau=s-1$. $R(\tau)$ is the self-adjusted range of the cumulative sums and $R S(\tau)$ is the self-rescaled self-adjusted range, which is the quantity of our interest.

Feller 25 has proved that the asymptotic behavior for the expectation value of any independent random process with finite variance is given by

$$
\lim _{\tau \rightarrow \infty} \mathrm{E}\left[\tau^{-\frac{1}{2}} R S(\tau)\right]=\sqrt{\pi / 2}
$$

The combination $R(\tau) / S(\tau)$ has a better sampling stability than $R(\tau)$, in the sense that the relative deviation of $R S$, defined as $\Delta R S(\tau)=\sqrt{\operatorname{Var}[R S(\tau)]} / \mathrm{E}[R S(\tau)]$, is smaller [26]. For an independent Gaussian process the limiting standard deviation is

$$
\lim _{\tau \rightarrow \infty} \operatorname{Var}[R S(\tau)]=\sqrt{\pi^{2} / 6-\pi / 2} \approx 0.2723 .
$$

On the other hand Hurst had found empirically that many time series of natural phenomena are described by the scaling relation

$$
R S(\tau) \propto \tau^{H}
$$

where $H$ differs significantly from $1 / 2$. In the context of fractional Brownian motion [24,26] a Hurst exponent of $H=1 / 2$ corresponds to the vanishing of correlations between past and future spatial increments in the record. For $H>1 / 2$ one has persistent behavior, that means a positive increment for some time in the past will on the average lead to a positive increment in the future (if the increments are distributed symmetrically around zero). Correspondingly the case of $H<1 / 2$ denotes antipersistent behavior.

Thus almost all long run correlations in the stochastic process should show up in deviations from the asymptotic (3), (4).

Furthermore, Mandelbrot and Wallis have demonstrated that the value of the asymptotic prefactor $\sqrt{\pi / 2}$ is not robust with respect to short run statistical dependence 26]. This value can be arbitrarily modified by cyclic components in the random process. The superposition of a white noise (with zero mean and unit variance) and a purely periodic process, for instance, leads to an asymptotic value of $\sqrt{\tau \pi / 2}(1+A / 2)^{-1 / 2}$, with $A$ being the amplitude of a sine wave. Moreover, the transient to the asymptotic is not smooth, but typically shows a series of oscillations, resembling the case of a purely oscillatory process 26].

Therefore the $R S$ statistic is perfectly suited to analyze a stochastic process for correlations on all scales.

In the following section several types of PRNGs will be used to generate $U(0,1)$-distributed random numbers $\xi_{t}$. The sequence of $\xi_{t}$ will then be analyzed according to the $R S$ statistic. It will be demonstrated that various PRNGs produce sequences of numbers that show deviations from the asymptotic behavior (3), (4). Moreover, it is found that for finite lags $\tau$ the value of $R S(\tau)$ differs significantly between the tested PRNGs being indicative of short range correlations. This way it is possible to obtain a complete "fingerprint" of correlations of a PRNG and to measure their intensity as a function of the lag.

\section{RANDOM GENERATORS}

Because of the vast number of different PRNGs currently employed in simulations only a small fraction can be selected in this work. 
The generators of the first group, labeled G1 to G7, are included as they are in general use - either because of traditions, because they are recommended in popular books, or because they can be found in many commercial software packages. Some of them have documented defects (G1,G2,G3,G5). These are considered here to study how their deviations show up in the $R S$ statistics. The generators in the second group, G8 to G11, have been proposed recently to match also future requirements on period length and quality. But there is little documented experience about their behavior in physical simulations. As there are many good reviews and books on the various generation methods and the performance in the standard tests [3, 5, 7,8,27, 29] only a brief outline of the considered algorithms is given in the next section.

\section{A. Generation Methods}

Most of the commonly used PRNGs are based on the linear congruential method. In general a multiple recursive generator of order $k$, denoted by $\operatorname{MRG}\left(a_{1}, \ldots, a_{k} ; c ; m\right)$, is based on the $k$ th-order linear recurrence

$$
x_{n}=\left(a_{1} x_{n-1}+\cdots+a_{k} x_{n-k}+c\right) \bmod m,
$$

where the order $k$ and the modulus $m$ are positive integers and the coefficients are integers in the range $\{-(m-1), \ldots, m-1\}$. The numbers $x_{n}$ of the sequence are then scaled to the interval $(0,1)$ by $u_{n}=x_{n} / m$.

The special case, where $k=1$, is the well-known linear congruential generator $\operatorname{LCG}(a ; c ; m)$ introduced by Lehmer [30], or in the homogeneous case, $c=0$, the multiplicative linear congruential generator, denoted by $\operatorname{MLCG}(a ; m)$. It can be shown that a recursion of order $k$ with a non-zero constant $c$ is equivalent to some homogeneous recurrence of order $k+1$ [5,28]. All congruential generators show a pronounced lattice structure. That means, if $n$ subsequent numbers are used to form vectors in the $n$-dimensional space all points that can be generated within the period lie on a family of equidistant parallel hyperplanes [6]. Tables with good choices for the constants can be found in recent reviews [3, 28,31,32.

A lagged Fibonacci generator, $\operatorname{LF}\left(l_{1}, \ldots, l_{k} ; m ; \circ\right)$, with $k$ lags is obtained for $c=0$ and $k$ coefficients $a_{i}$ being set to unit modulus, the others being set to zero,

$$
x_{n}=\left(x_{n-l_{1}} \circ \cdots \circ x_{n-l_{k}}\right) \bmod m .
$$

The binary operator $\circ$ is usually either addition or subtraction.

The Linear feedback shift register or Tausworthe method, $\operatorname{LFSR}(p, q)$, generates a sequence of binary digits (bits) $b_{n}$ from the recurrence relation

$$
b_{n}=b_{n-p} \oplus b_{n-q}
$$

where the exclusive-or operation $\oplus$ is equivalent to a bitwise addition modulo two 8,33 . A sequence of pseudorandom numbers is then obtained by taking an appropriate number of consecutive bits to form an integer number.

Generalized feedback shift register generators [34], denoted by $\operatorname{GFSR}\left(l_{1}, \ldots, l_{k} ; m\right)$, which can be considered as a generalization of the Tausworthe generator, are related to the lagged Fibonacci method, but use the exclusive-or operation instead of the arithmetic operators to combine computer words $w$

$$
w_{n}=w_{n-l_{1}} \oplus \cdots \oplus w_{n-l_{k}}
$$

A generator of this type with two lags (103 and 250) has been made popular by Kirkpatrick and Stoll and is known as R250 35, 36] (see also [9]). A particular realization with four lags has been given by Ziff [37] (for test results see 18 21]). A recently proposed special variant with huge period is the twisted GFSR generator, TGFSR [17].

The multiply-with-carry generator, denoted by $\operatorname{MWC}\left(a_{1}, \ldots, a_{k} ; c ; m\right)$, is defined by the recurrence relation

$$
\begin{aligned}
x_{n} & =\left(a_{1} x_{n-1}+\cdots+a_{k} x_{n-k}+c_{n-1}\right) \bmod m, \\
c_{n} & =\left(a_{1} x_{n-1}+\cdots+a_{k} x_{n-k}+c_{n-1}\right) \operatorname{div} m .
\end{aligned}
$$

The div denotes an integer division. Here, in contrast to the MRG a carry (or borrow) $c_{n}$ is propagated to the next iteration step.

Special cases of the MWC are the the add-withcarry, $\mathrm{AWC}\left(l_{1}, l_{2} ; m\right)$, and the subtract-with-borrow, $\operatorname{SWB}\left(l_{1}, l_{2} ; m\right)$, generators, which are obtained by setting two coefficients $a_{i}$ to unit modulus and all others equal to zero [14,38]. This basically results in a LF generator with two lags, but with an extra addition of a carry

$$
\begin{aligned}
x_{n} & =\left(x_{n-l_{1}}+x_{n-l_{2}}+c_{n-1}\right) \bmod m, \\
c_{n} & =\left[x_{n-l_{1}}+x_{n-l_{2}}+c_{n-1} \geq m\right] .
\end{aligned}
$$

In the case of an $\mathrm{AWC}$ the bracket indicates the value of the carry which is equal to 1 if the inequality is true, and equal to 0 otherwise. In the case of an SWB the addition operations accordingly have to be replaced by subtractions and the borrow is equal to 1 if the result of the subtractions becomes negative. These generators can produce much longer periods than the underlying LF generators, but have a bad lattice structure in dimension $l+1,(l$ being the larger of the lags $)$ 3, 5, 39. 3.

The subtraction method, $\operatorname{SUB}(c ; m)$, is based on a simple arithmetic sequence

$$
x_{n}=\left(x_{n-1}-c\right) \bmod m .
$$

This method is not suitable by itself, but it may be included in combination generators $[7,40]$.

The multiplicative quadratic congruential method, MQC [4]8], the cryptographic BBS [41] and DES [42] generators, or the inversive congruential generator, $\mathrm{ICG}[43]$ 
are only mentioned for completeness, as these have received considerable theoretical attention recently. These new methods have promising features, but the generators are currently not in common use as there is little practical experience with them.

In general the PRNGs with several lags require an initial set of seeds $x_{1}, \ldots, x_{k}$ the number of which is determined by the largest lag $k$. While most generators do not require a special initialization procedure care has to be taken with the GFSR generators. Here an improper selection of the seeds can severely affect the quality of the sequence of PRNs 44. Often a congruential generator is used to generate the initial state.

Tausworthe and LFSR generators which are based on the theory of primitive trinomials form unfavourable structures similar to the lattice structure of LCGs and have bad statistical properties [16,29]. Such simple generators should be avoided and combined generators should be used instead.

There is strong empirical support that the combination of different pseudorandom sequences in general leads to an improved statistical behavior [4, 45. The two wellknown methods are the shuffling of one sequence with another or with itself [4] 8 or the combination by modular addition [28,32. Hybrid generators based on the first method are still not well understood from the theoretical viewpoint 3, 5]. The latter method is better understood and is suited to obtain very long periods. Adding two sequences modulo the modulus of either of them the period obtained is the least common multiple of the component periods. Generators based on such combination methods currently provide us with the "best" PRNs. Many different kinds of combined generators have been proposed, see Refs. 44,5,7, 14 -16, 28, 32, 40 and references given therein.

Another common method which can lead to an improvement of a generator is a decimation strategy, that means a number of PRNs is thrown away before the next random number is delivered. This approach is taken for instance in the RANLUX generator 46, 47 which significantly improves the defective SWB generator RCARRY [7,38]. But neither shuffling nor the decimation method may be desirable if speed considerations are very important (see Appendix B for timing results).

In the following the generators subjected to the $R S$ statistical analysis are described in brief.

\section{B. Tested Generators}

G1 is the well-known MLCG $\left(7^{5} ; 2^{31}-1\right)$, which has been proposed as the "mimimal standard' against which all other generators should be judged [27,31,48. It is also known as GGL [31], CONG [9], ran0 [42,49], SURAND (IBM computers), RNUM (IMSL library), or RAND (MATLAB software). It has the serious drawback of a short period, $2^{31}-1$, and a pronounced lattice structure in low dimensions. Multiplier and modulus are not the optimal choice considering several figures of merit, see for instance [3]. This generator should only be considered as a toy for experimenting with new test methods like all other simple congruential and LFSR generators.

G2 is identical to G1, but additionally Bays-Durham shuffling in a table of size 32 is used to improve the low-order serial correlations. Here the implementation ran1 of Ref. 42, 49 has been applied. It is included in this test to show the influence of shuffling on the $R S$ statistic.

G3 is a $\operatorname{LF}\left(55,24 ; 2^{31} ;-\right)$ generator which has a period of $2^{55}-1$. It has been devised by Mitchell and Moore in 1958 and is described by Knuth [4] (originally using an add operation). This generator (a version of which is implemented in 42 as ran3) is reported to have significant correlations on the bitlevel and to fail several physical tests $11,18,21$. It is included to demonstrate the effect of short range correlations on the $R S$ statistic.

G4 is a modification of the above generator G3. If a decimation strategy is used, that means, if only every $k$-th number of the sequence is used, the generator passes all of the physical tests in Ref. [18 20 (for $k=2$ and $k=3$ ). In this work only the case of $k=3$ is considered.

G5 ist the GFSR(250,103,2 $\left.2^{32}\right)$ generator R250 proposed by Kirkpatrick and Stoll [35.36]. It has a period of $2^{250}$. While this generator performs well in the standard statistical tests it is reported to fail several physical tests [9,18 21.

G6 The combination generator RANMAR proposed by Marsaglia and Zaman [7, 40 has a period of about $2^{144}$. It is based on the subtraction modulo $2^{24}$ of a simple arithmetic sequence

$\operatorname{SUB}\left(7654321 ; 2^{24}-3\right)$

and a subtractive Fibonacci generator

$\operatorname{LF}\left(97,33 ; 2^{24} ;-\right)$

The initial state is generated by another combination of $\operatorname{LCG}(53 ; 1 ; 169)$ and a multiplicative threelag Fibonacci sequence. The implementation of James [7] tested here is in wide-spread use and has been recommended as a "universal generator".

G7 combines the two congruential sequences

$$
\operatorname{MLCG}\left(40014 ; 2^{31}-85\right)
$$
and

MLCG $\left(40692 ; 2^{31}-249\right)$

by modular addition and applies an additional shuffling in a table of 32 entries. The period is approximately $2^{62}$. This algorithm has been invented by L'Ecuyer 32] and implemented by James [7] (called RANECU). The additional shuffling has been added in the version ran2 of Press et al. 42, 49. Many recommendation for the improvement for instance 
of the speed) of the later version have been given by Marsaglia and Zaman 14. They reported that this generator passes all standard tests. Because of its popularity the implementation of Ref. 442,49] has been used in the following $R S$ analysis.

G8 is the recently proposed PRNG mzran13 of Marsaglia and Zaman. It combines

LCG(69069, 1013904243; $\left.2^{32}\right)$

and

$\operatorname{SWB}\left(2,3 ; 2^{32}-18\right)$

by modular addition and has a period of about $2^{125}$ 14. Although the published program takes advantage of the inherent modulo $2^{32}$ arithmetic of modern CPU's it can easily be made portable to CPUs with any larger word size by using bit-masks.

G9 This is a composite generator of L'Ecuyer [15] based on the modular addition of the sequences of

$$
\operatorname{MRG}\left(0,63308,-183326 ; 0 ; 2^{31}-1\right)
$$
and

$$
\text { MRG(86098, 0, -539608; 0; } \left.2^{31}-2000169\right) \text {. }
$$

It has a very long period of about $2^{205}$ and a lattice structure with theoretically better properties than G7 15.

G10 This generator is the maximally equidistributed three-component Tausworthe generator taus88 developed by L'Ecuyer [16] with a period of approximately $2^{88}$.

G11 The twisted GFSR generator TT800 proposed by Matsumoto and Kurita 17] has a huge period of $2^{800}-1$ and is reported to have excellent equidistribution properties up to a dimension of 25 . This generator is recommended in [3]. The tested version includes Matsumoto's code change of 1996 which improves the lower bit correlations.

\section{DESCRIPTION OF THE TEST AND RESULTS}

\section{A. The Test Setup}

A few additional words have to be said about the generation of the initial seeds for the PRNGs. As these are (possibly) the only truly random part when generating pseudorandom numbers some care should be taken.

The following method has been applied, as it corresponds to a common way random generators are used in practice:

The initial seed is calculated from a combination of some obviously truly random events, such as the time and the date when the program is started, several system specific (unique) process identifiers, and the processor clock state. For this initial seed a sequence of $10^{9}$ to $10^{10}$ random numbers is generated and analyzed according to
(1). Then for some new random seed another sequence is obtained and analyzed.

This procedure has been iterated until the statistical error for the average of $R S(\tau)$ was considered small enough. For each of the generators this amounted to $10^{11}$ to $10^{12}$ generated PRNs.

As this approach does not ensure that the generated substreams are disjoint it might look safer to split the period into disjoint parts. This could be done for almost all generators, but there are several cases known where these (typically) equidistantly spaced seeds introduce even worse correlations [5]. One should also bear in mind that for the long period generators there is only a very small probability that, for instance, ten or twenty sequences of $10^{10}$ numbers selected by a random seed are not disjoint (of course the period of the "toy" generators is exhausted immediately).

In the case of generators requiring more than one seed one initial seed has been generated and mixed into the default seeds of the original source code. For instance, the 25 published seeds that define the state of the TGFSR generator G11 have all been exclusive-or-ed with a new random seed every time a new sequence has been generated.

All calculations necessary to evaluate the $R S$ statistic have been performed in double precision using IEEE 754 standard floating point arithmetics.

The number of PRNs generated in the test of each generator is comparable to the number of random variates typically required in a nowadays high precision MonteCarlo simulation. Such a number may seem large for a mere test, but it comprises the current state of the art in research fields like percolation, random walks, diffusion limited aggregation, and many others [9, 11, 13. Considering the speed of the advances in computer technology much larger simulations will be in reach within the next few years posing increased demands on precision to the PRNGs. Correspondingly the stringency of the empirical tests has to increase too.

In the following section it will be shown that several current thought-to-be-reliable PRNGs show pronounced correlations in the $R S$ statistic. This does not mean that a large scale simulation inevitably produces erroneous results with such a PRNG, but it just means that in some types of simulations deviations are not unlikely if high precision is required. Moreover, the main purpose of this paper is to demonstrate that the $R S$ statistic is a candidate to enrich the toolbox of empirical tests for random number generators.

\section{B. Analysis of the RS Data}

In Fig. 1 the diagram of $\log R S(\tau)$ versus $\log \tau$ is shown for all tested random generators. $R S(\tau)$ has been calculated for all powers of two in the range from $\tau=2$ up to $\tau=2^{23} \approx 8 \times 10^{6}$ as indicated by the dots. After 


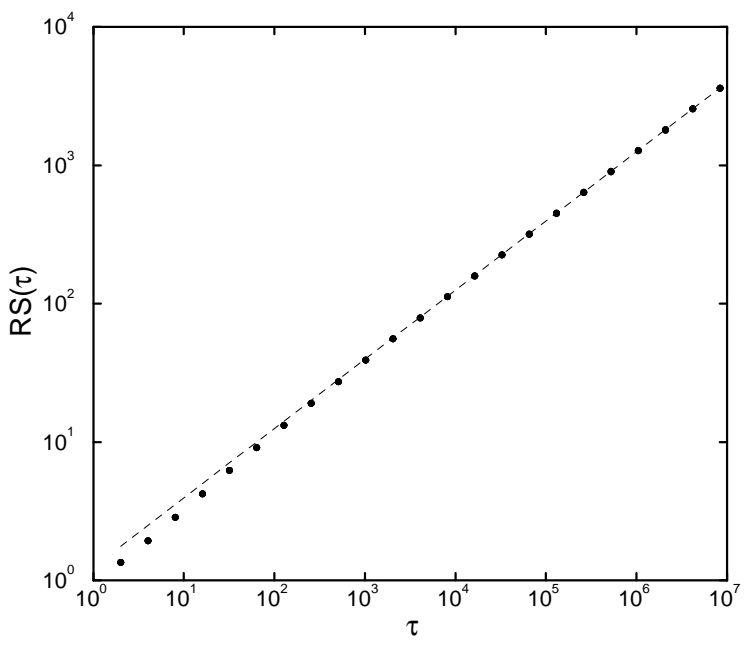

FIG. 1. Double-logarithmic plot of the numerical data $(\bullet)$ of $R S(\tau)$ for all PRNGs. On this scale the results for the various PRNGs are indistinguishable. The asymptotic $\sqrt{\tau \pi / 2}$ behavior is indicated by the broken line.

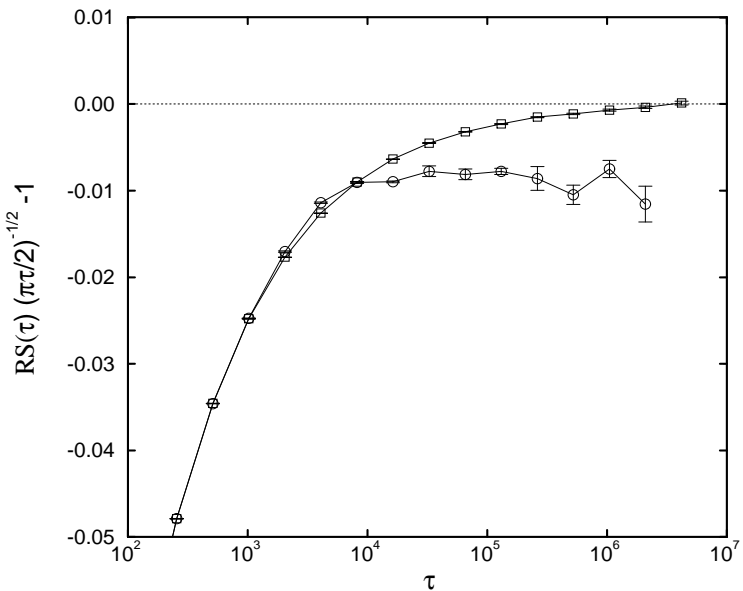

FIG. 2. Semi-logarithmic plot of $R S(\tau)(\pi \tau / 2)^{-1 / 2}-1$ for the pseudorandom number generators G1 (०) and G9 ( $\square)$. The lines are intended as a guide to the eye.

a transient behavior for lags smaller than $\tau \approx 10^{4}$ the asymptotic law (3) applies almost perfectly. But on this scale the results for the various PRNGs are indistinguishable for all lags.

To resolve differences between the PRGNs it is convenient to remove the asymptotic trend. In Fig. 2 the reduced function $R S(\tau)(\pi \tau / 2)^{-1 / 2}-1$ is displayed for a generator with known correlations, G1 (o), and the combination generator G9 $(\square)$. On this scale of magnification it can be seen that the simple LCG spectacularly fails to approach the expected asymptotic. The relative deviation becomes as large as 1\% corresponding to a reduced asymptotic prefactor (which appears to be approximately 1.243 instead of $\sqrt{\pi / 2}=1.253$ ). For comparison the data for the highly reliable composite MRG G9 are shown. In this case the asymptotic expectation value is approached smoothly. Due to the large statistical ensemble the error

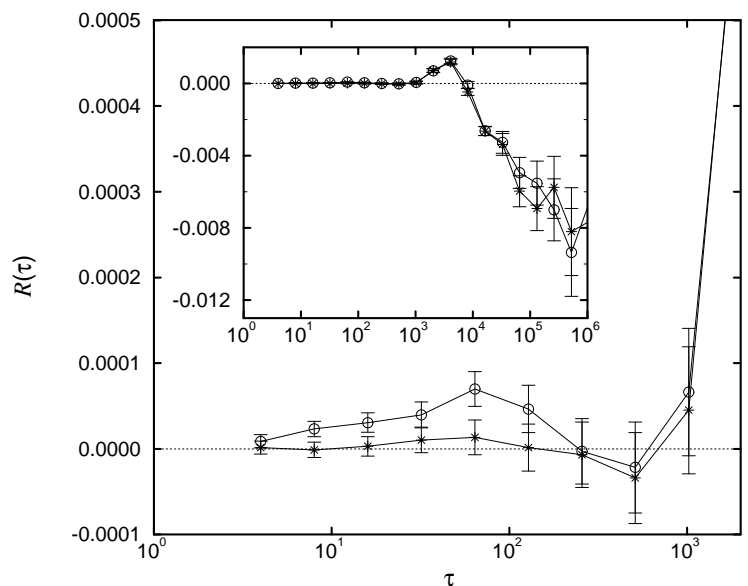

FIG. 3. $\mathcal{R}(\tau)$ versus $\tau$ for G1 (o) and G2 (*) illustrating the effect of a shuffle table. The inset shows a larger range of $\tau$.

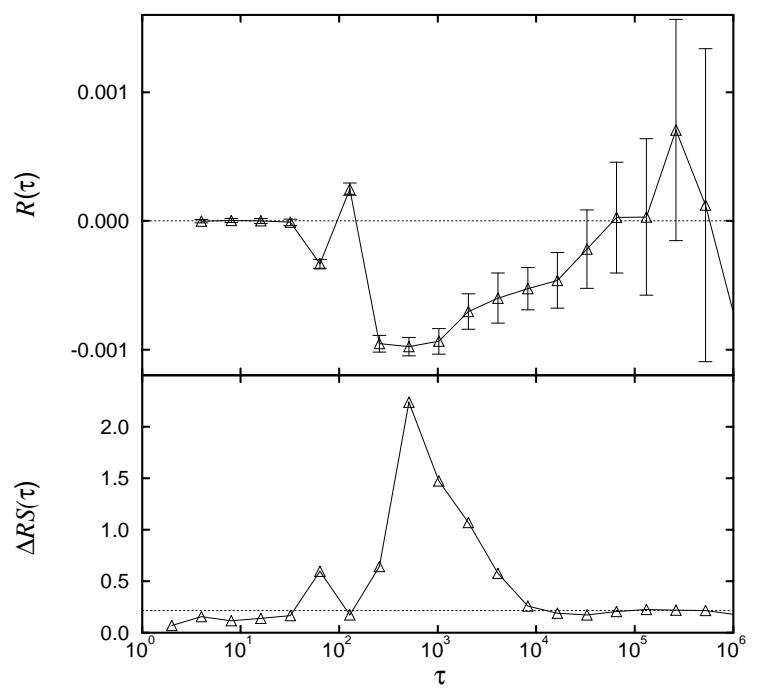

FIG. 4. Upper figure: $\mathcal{R}(\tau)$ versus $\tau$ for the LF generator G3 $(\triangle)$. Lower figure: Drastic deviations from the asymptotic value (dotted line) are also visible in $\Delta R S(\tau)$.

bars appear as single lines.

The distribution of the numerical $R S$ values for all lags is well described by the slightly right-skewed asymptotic density as given by Feller [25]. The half width of the error bars for the estimate of the mean (in this and the following figures) is given by two standard deviations according to the asymptotic analytical result (4). This corresponds to a confidence level of about $95 \%$. The numerical results for the mean together with the standard deviation of the mean are tabulated in Appendix A for all generators of this test.

As with several other test statistics where only the asymptotic distribution is available one is limited to compare the generators. Comparing the estimate of the mean for finite lags with the asymptotic expectation one could always enforce a rejection of a generator if the the number of samples is sufficiently increased. In the following a method is described which facilitates the comparison of 


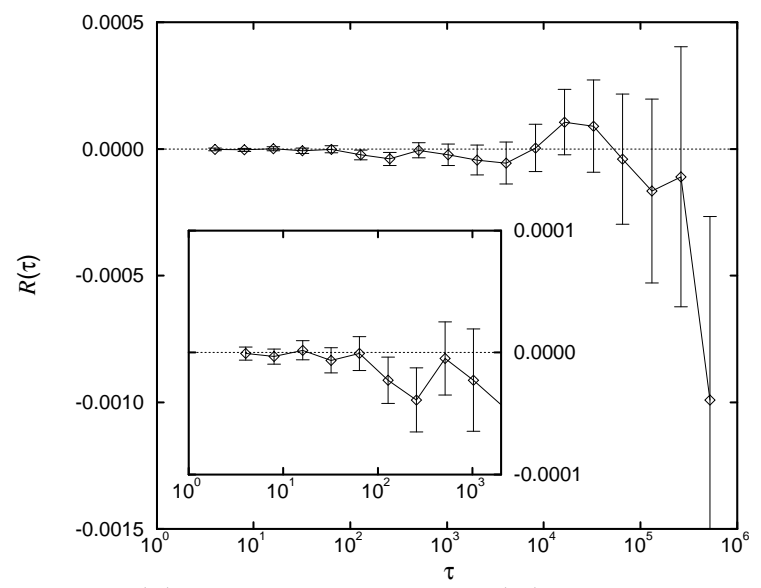

FIG. 5. $\mathcal{R}(\tau)$ for the generator G4 $(\diamond)$. Inset: Magnified view for small lags $\tau$.

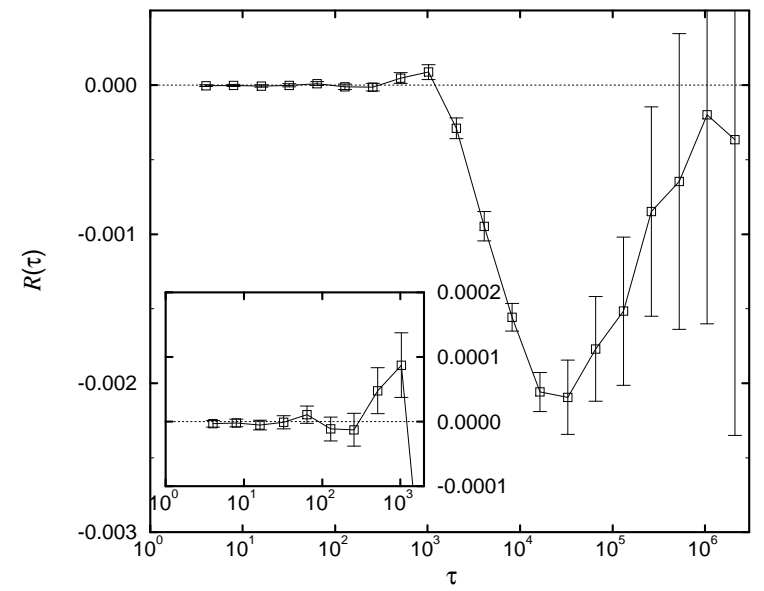

FIG. 6. $\mathcal{R}(\tau)$ for the GFSR generator G5 (+). Inset: Magnified view for small lags $\tau$.

$R S(\tau)$ for the different generators.

It can be safely assume that the asymptotic limit is approached smoothly with increasing $\tau$. Therefore any apparent local and non-monotone structure in the transient will be indicative of correlations. Analyzing the functional form of the transient a simple and smooth interpolation can be found which gives an accurate approximation for all lags within a range of more than 6 orders of magnitude. The transient of $R S(\tau)$ can be parametrized by

$$
\begin{gathered}
\mathcal{R}(\tau) \equiv\left(\frac{R S(\tau)}{\sqrt{\pi \tau / 2}-\alpha}-1\right)-\left(\frac{1}{\arctan \beta \tau}-\frac{2}{\pi}\right) \\
+\gamma e^{-\delta \tau^{\varepsilon}} .
\end{gathered}
$$

Using only two parameters $\alpha, \beta$ the first two terms suffice to approximate the transient with a relative precision of $\approx 10^{-5}$ for all lags larger than $\tau=32$. The last term in (13) has been introduced to approximate the transient for lags as small as $\tau=4$. The coefficients have been obtained from a numerical adjustment using the mean values obtained from the stronger generators G8, G9, and

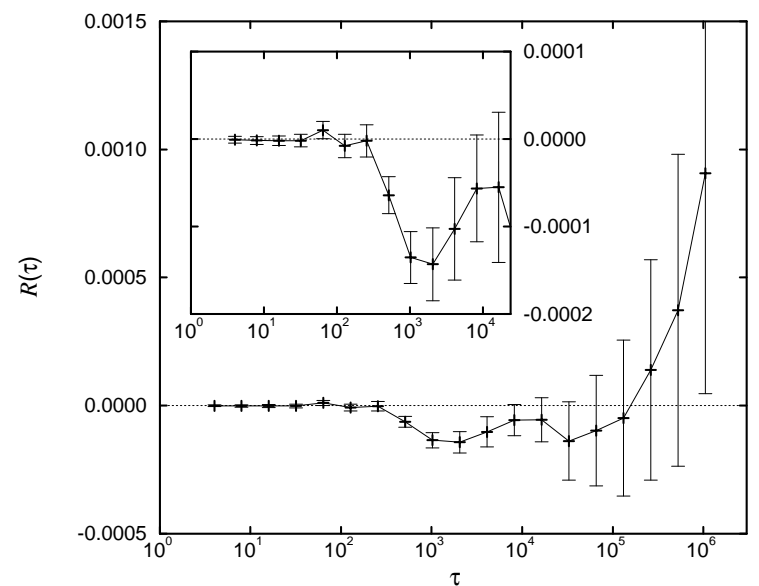

FIG. 7. $\mathcal{R}(\tau)$ for the combination generator G6 $(\times)$. Inset: Magnified view for small lags $\tau$.

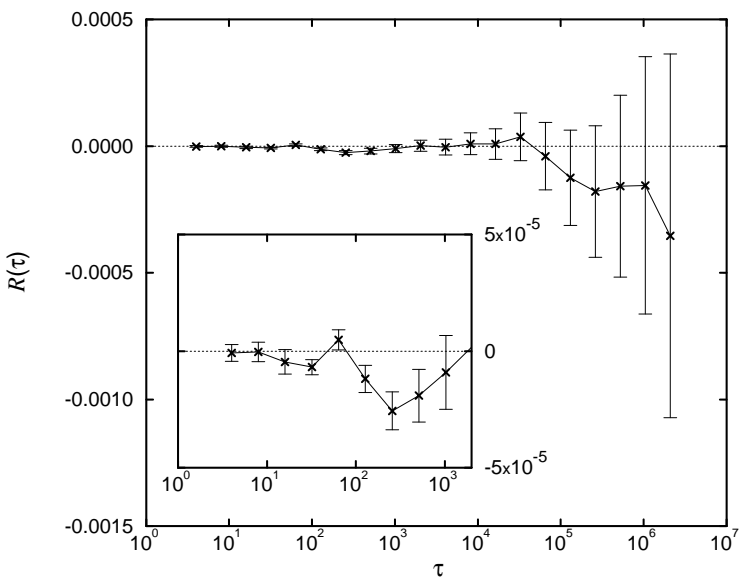

FIG. 8. $\mathcal{R}(\tau)$ for the combination generator G7 $(\square)$. Inset: Magnified view for small lags $\tau$.

G10 with $\tau$ in the range from 4 to $2^{14}$. In this range the individual results agree to a high precision. The values of the coefficients in (13) used in the following are

$\alpha \approx 1.0319941 \quad \gamma \approx 0.10516938 \quad \varepsilon \approx 0.61775533$

$\beta \approx 0.42091184 \quad \delta \approx 0.90187633$

The smooth interpolation $\mathcal{R}(\tau)$ of the transient now allows an unbiased comparison of the various PRNGs. As the expectation values for finite $\tau$ are not known the approximation (13),(14) is used instead. The generators can now be compared with the approximate transient. This approach has been found to be superior to comparing the generators individually. In particular the influence of statistical fluctuations of the mean are minimized compared to a pairwise comparison of the generators at a given value of $\tau$. In the following it will become clear that the important point is not to have a precise approximation of the transient for truly random numbers. The detection of a deviation is insensitive to the exact form of the approximation: in all cases a defect showed up as a pronounced wiggle in $R S(\tau)$ around the monotone transient. Therefore the subtraction of any monotone and 


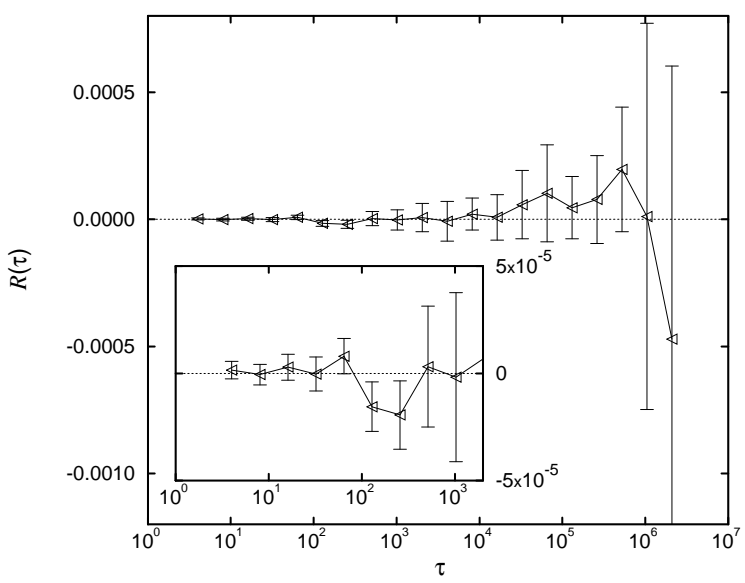

FIG. 9. $\mathcal{R}(\tau)$ for the combination generator G8 $(\triangleleft)$. Inset: Magnified view for small lags $\tau$.

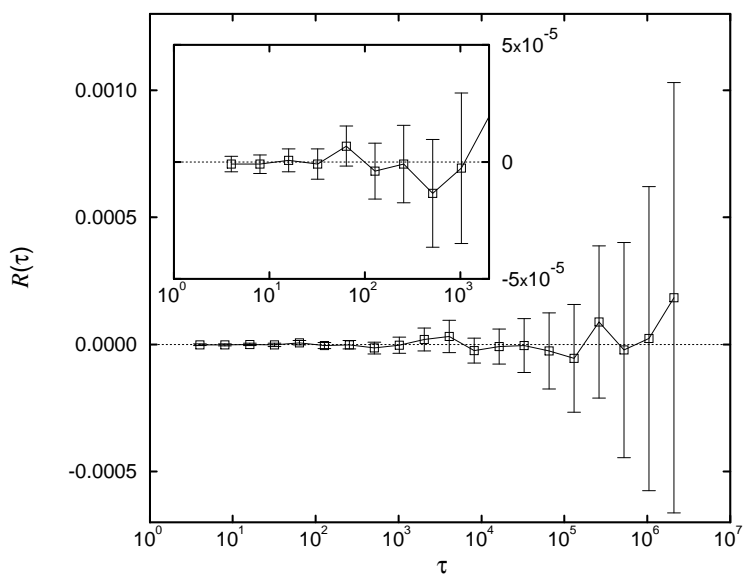

FIG. 10. $\mathcal{R}(\tau)$ for the combination generator G9 ( $\square)$. Inset: Magnified view for small lags $\tau$.

slowly varying function would suffice to reveal a characteristic "fingerprint" of correlations in the PRNG. All systematic deviations of $\mathcal{R}(\tau)$ from zero are indicative of the presence of correlations and the amplitude at lag $\tau$ can be considered as a measure of the strength of correlations for the given lag. Hence the various PRNGs can be compared quantitatively.

\section{Discussion of the Results}

In Fig. 3 the semi-logarithmic plots of $\mathcal{R}(\tau)$ versus $\log \tau$ for the toy generators G1 (o) and G2 $(*)$ are shown for lags between 4 and $2^{21} \approx 2 \times 10^{6}$ (inset). Serious deviations are evident for lags larger than $10^{3}$. Magnifying on the vertical axis by a factor of 25 the plot of $\mathcal{R}(\tau)$ reveals deviations also at small lags (main figure). In generator G2 additional shuffling in a small table has been introduced to improve low order serial correlations of generator G1. For lags up to $\tau \approx 128$ the deviations are indeed strongly reduced. As expected there is no improvement for lags which are much larger than the size of the shuffling table.

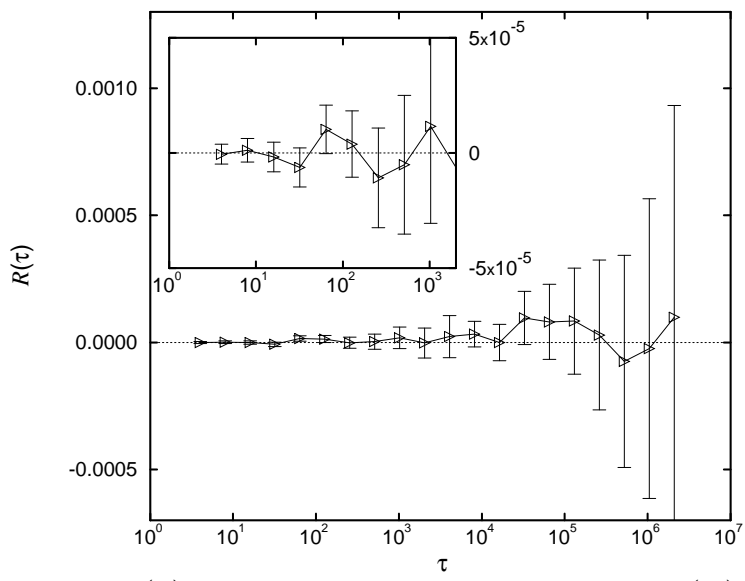

FIG. 11. $\mathcal{R}(\tau)$ for the combination generator G10 $(\triangleright)$. Inset: Magnified view for small lags $\tau$.

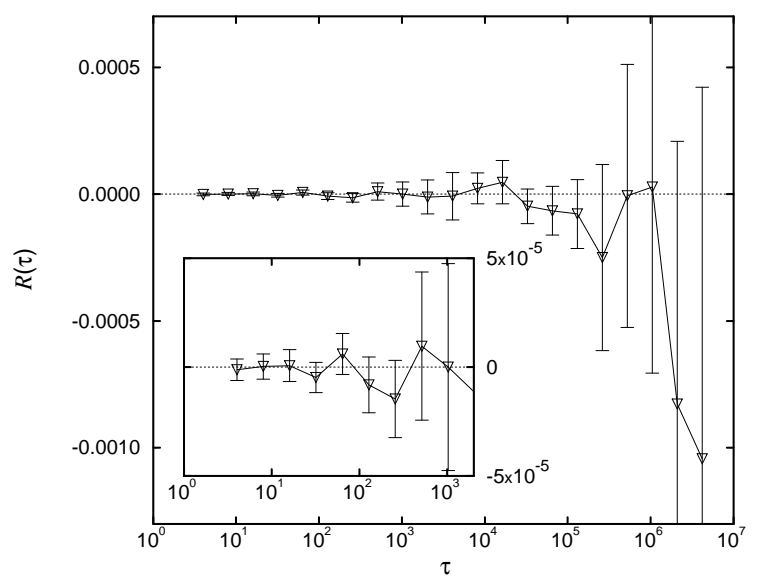

FIG. 12. $\mathcal{R}(\tau)$ for the TGFSR generator G11 $(\nabla)$. Inset: Magnified view for small lags $\tau$.

In Fig. 0 the results for the lagged Fibonacci generator G3 $(\triangle)$ are shown. This generator is known to fail several tests (see Ref. 18 21 and appendix Q). It is reassuring to see that the $R S$ statistic easily reveals the onset of disastrous correlations at $\tau$ corresponding to the larger lag of the generator $(l=55)$. The deviations show up as a crossover of $\mathcal{R}(\tau)$ (upper figure) to a "shifted asymptotic" reflecting a modified asymptotic prefactor. This gives evidence to the presence of some strong cyclic components in the pseudorandom process of G3. This is the only generator in this test showing also deviations of $\Delta R S(\tau)$ from the asymptotic value (Fig. 4 lower graph).

If a decimation strategy with $k=3$ is applied, corresponding to generator G4 $(\diamond)$, the correlations are strongly suppressed (Fig. 5).

The GFSR generator G5 (Fig. 6) uses larger lags than G3 shifting the onset of correlations to larger $\tau$. The magnitude of the deviation is even twice as large as that of generator G3. These dramatic deviations are obviously indicators for the poor behaviour of G5 in some MonteCarlo (MC) simulations [18].

Pseudorandom numbers of much better quality are expected from combination generators which can overcome 
the weakness of generators which are structurally too simple.

In Fig. 7 the performance of the popular combination generator G6 $(+)$ can be estimated. When $\tau$ is somewhat larger than the lags of the LF component of the generator significant deviations in $\mathcal{R}$ are observed (similar to G3 and G5). These are presumably due to the deficient LF component of the composite generator. But compared to G5 the deviation is about 10 times smaller. For the time being there are no documented failures in physical simulations that use this generator 19]. But comparing Fig. 7 with Figs. 4, 6 one can conclude that deviations in MC simulations are not implausible if higher precision is demanded.

PRNGs which are as fast, but which have better longrange properties are discussed in the following. In the next figure, Fig. 8, the results for the combined congruential generator $\mathrm{G} 7(\times)$ are shown. Compared to the previous generators the amplitude of the deviations is drastically decreased. But for lags in the range $\tau=2^{5}$ to $2^{9}$ a structure being indicative of correlations can be resolved (see inset of figure and Tab. III) on a high level of significance. Although G7 is doubtlessly one of the better generators within this test it should be immediately evident that it cannot come up to the expectations of Press and Teukolsky [42,49 to provide perfect random numbers (within the limits of its floating point precision). Thus their proposed "practical" definition of perfect should at least be put into perspective.

Random numbers of much better quality (at least in the RS statistics) are generated by the recently proposed composite generators G8 to G11. For all lags in the range $2^{2}$ to $2^{21}$ there are no significant differences. These four PRNGs are based on four different generation methods. Generator G8 applies a combination of generators with different algebraic structure while the two-component MRG G9 and the three-component Tausworthe generator G10 combine generators of the same class. Finally, G11 is a TGFSR generator which distinguishes itself by an extraordinary long period [51]. The fact that four generators of completely different algebraic structure and with theoretically favourable properties give consistent results reassures that the observed deviations of the other generators are indicators of real defects.

It should be noted that $R S(\tau)$ necessarily has been sampled on a coarse grid on the logarithmic scale. Therefore it is possible that several types of correlations which would have shown up as a narrow structure have not been recognized. Nevertheless the observed deviations are intriguing.

\section{CONCLUSIONS}

The sensitivity for correlations on all scales and the robustness predestinates the $R S$ statistic as a tool to catch up defects in pseudorandom number generators. A prac- tical method has been described which makes it easy to obtain a characteristic fingerprint of the correlations in a pseudorandom sequence. The deviations can be described quantitatively and the performance of generators for some given range of lags can be compared.

To illustrate the capability of the $R S$ statistical analysis several popular generators have been subjected to an extensive test. The randomness of all tested PRNGs whith known defects could be refuted. Moreover deviations in several generators which are thought to be reliable have been quantified. Thus the $R S$ analysis has to be considered more stringent than many of the previously suggested tests in the sense that more generators fail it.

The selection of a PRNG for a specific simulation depends on the required level of precision and on the range of the correlations which may have an impact on the quantity of interest - although this often cannot be assessed in advance. But no generator showing a performance inferior to another generator in several tests should be used any longer if it doesn't even distinguish itself at least by speed. Weak correlations in a current state-of-the-art generator (like some of this test) can lead to erroneous results in a tomorrow high-precision calculation.

\begin{tabular}{|c|c|c|c|}
\hline$\tau$ & G1 & G2 & G3 \\
\hline$\overline{2^{2}}$ & $\left.8.836(3.84) 10^{-6}\right]_{2}$ & $1.572(3.84) 10^{-6}$ & $-1.228(6.61) 10^{-6}$ \\
\hline $2^{3}$ & $2.331(0.45) 10^{-5}{ }_{5}$ & $-1.166(4.50) 10^{-6}$ & $3.183(7.76) 10^{-6}$ \\
\hline $2^{4}$ & $3.075(0.57) 10^{-}$ & $2.972(5.68) 10^{-6}$ & $1.010(9.79) 10^{-6}$ \\
\hline $2^{5}$ & $3.969(0.75) 10^{-}$ & $1.045(0.75) 10^{-5}$ & $-1.026(1.29) 10^{-5}$ \\
\hline $2^{6}$ & $6.990(1.01) 10^{-}$ & $1.348(1.01) 10^{-5}$ & $-3.324(0.17) 10^{-}$ \\
\hline $2^{7}$ & $4.659(1.38) 10^{-}$ & $1.593(13.8) 10^{-6}$ & $2.483(0.24) 10^{-}$ \\
\hline $2^{8}$ & $-2.911(19.0) 10^{-6}$ & $-6.774(19.0) 10^{-6}$ & $-9.531(0.33) 10$ \\
\hline $2^{9}$ & $-2.170(2.65) 10^{-5}$ & $-3.392(2.65) 10^{-5}$ & $-9.763(0.35) 10^{-}$ \\
\hline $2^{10}$ & $6.632(3.71) 10^{-5}$ & $4.517(3.71) 10^{-5}$ & $-9.344(0.49) 10^{-}$ \\
\hline $2^{11}$ & $7.057(0.52) 10^{-4}$ & $7.230(0.52) 10^{-}$ & $-7.032(0.69) 10^{-}$ \\
\hline $2^{12}$ & $1.249(0.07) 10^{-}$ & $1.192(0.07) 10^{-}$ & $-5.987(0.98) 10^{-}$ \\
\hline $2^{13}$ & $-8.803(9.78) 10^{-}$ & $-4.659(0.98) 10^{-}$ & $-5.266(0.82) 10^{-}$ \\
\hline $2^{14}$ & $-2.627(0.13) 10^{-}$ & $-2.637(0.13) 10^{-}$ & $-4.612(1.08) 10$ \\
\hline $2^{15}$ & $-3.263(0.31) 10^{-}$ & $-3.378(0.31) 10^{-}$ & $-2.177(1.53) 10^{-4}$ \\
\hline $2^{16}$ & $-4.948(0.43) 10^{-}$ & $-5.969(0.43) 10^{-}$ & $2.702(21.5) 10^{-5}$ \\
\hline $2^{17}$ & $-5.529(0.61) 10^{-}$ & $-6.934(0.61) 10^{-}$ & $3.151(30.4) 10^{-5}$ \\
\hline $2^{18}$ & $-7.006(0.86) 10^{-}$ & $-5.760(0.86) 10^{-}$ & $7.069(4.30) 10^{-4}$ \\
\hline $2^{19}$ & $-9.363(1.22) 10^{-}$ & $-8.212(1.22) 10^{-3}$ & $1.232(6.08) 10^{-4}$ \\
\hline
\end{tabular}

TABLE I. The numerical values of $\mathcal{R}(\tau)$ are tabulated in columns for the generators G1, G2, G3. The value of one standard deviation $(\sigma)$ of the mean is given in parenthesis. If the deviation is larger than $2 \sigma$ the value is framed and the deviation in units of $\sigma$ is attached to the right.

\section{APPENDIX A: NUMERICAL RESULTS}

The numerical results for the mean of $\mathcal{R}(\tau)$, as depicted in previous figures, are reported in tables $[$ to IV. The value of one standard deviation of the mean is given in parenthesis. Values which differ from zero by more than two standard deviations are framed and the deviation in units of standard deviations is printed behind the box. 


\begin{tabular}{|c|c|c|c|}
\hline$\tau$ & G4 & G5 & G6 \\
\hline$\overline{2^{2}}$ & $-8.584(26.4) 10^{-7}$ & $-3.322(2.58) 10^{-6}$ & $-7.225(18.7) 10^{-7}$ \\
\hline $2^{3}$ & $-3.268(3.10) 10^{-6}$ & $-2.058(3.03) 10^{-6}$ & $-1.715(2.20) 10^{-6}$ \\
\hline $2^{4}$ & $1.845(3.92) 10^{-6}$ & $-5.343(3.82) 10^{-6}$ & $-1.889(2.77) 10^{-6}$ \\
\hline $2^{5}$ & $-6.375(5.15) 10^{-6}$ & $-1.010(5.03) 10^{-6}$ & $-1.744(3.64) 10^{-6}$ \\
\hline $2^{6}$ & $-7.679(69.4) 10^{-7}$ & $1.074(0.68) 10^{-5}$ & $1.019(0.49) 10^{-5}{ }_{2}$ \\
\hline $2^{7}$ & $-2.283(0.95) 10^{-5}{ }_{2}$ & $-1.135(0.93) 10^{-5}$ & $-7.898(6.71) 10^{-6}$ \\
\hline $2^{8}$ & $-3.893(1.31) 10^{-5}{ }_{2}$ & $-1.254(1.28) 10^{-5}$ & $-2.120(9.28) 10^{-6}$ \\
\hline $2^{9}$ & $-4.791(15.0) 10^{-6}$ & $4.778(1.78) 10^{-5}{ }_{2}$ & $-6.419(1.06) 10^{-5}$ \\
\hline $2^{10}$ & $-2.263(2.10) 10^{-5}$ & $8.746(2.49) 10^{-5}$ & $-1.354(0.15) 10^{-4}$ \\
\hline $2^{11}$ & $-4.342(2.94) 10^{-5}$ & $-2.890(0.35) 10^{-}$ & $-1.432(0.21) 10^{-}$ \\
\hline $2^{12}$ & $-5.510(4.14) 10^{-5}$ & $-9.464(0.49) 10^{-}$ & $-1.028(0.29) 10^{-4}$ \\
\hline $2^{13}$ & $4.312(46.7) 10^{-6}$ & $-1.557(0.05) 10^{-}$ & $-5.653(3.05) 10^{-5}$ \\
\hline $2^{14}$ & $1.065(0.64) 10^{-4}$ & $-2.058(0.07) 10^{-}$ & $-5.522(4.30) 10^{-5}$ \\
\hline $2^{15}$ & $9.044(9.09) 10^{-5}$ & $-2.094(0.12) 10^{-}$ & $-1.385(0.76) 10^{-4}$ \\
\hline $2^{16}$ & $-3.961(12.8) 10^{-5}$ & $-1.770(0.18) 10^{-3}$ & $-9.816(10.8) 10^{-5}$ \\
\hline $2^{17}$ & $-1.651(1.81) 10^{-4}$ & $-1.516(0.25) 10^{-3}$ & $-4.896(15.2) 10^{-5}$ \\
\hline $2^{18}$ & $-1.093(2.56) 10^{-4}$ & $-8.485(3.51) 10^{-4}$ & $1.387(2.15) 10^{-4}$ \\
\hline $2^{19}$ & $-9.906(3.62) 10^{-4}$ & $-6.466(4.96) 10^{-4}$ & $3.722(3.04) 10^{-4}$ \\
\hline $2^{20}$ & $-6.947(5.12) 10^{-4}$ & $-1.979(7.02) 10^{-4}$ & $9.075(4.30) 10^{-4}{ }_{2}$ \\
\hline
\end{tabular}

TABLE II. The numerical values of $\mathcal{R}(\tau)$ are tabulated in columns for the generators G4, G5, G6. See Tab. If for an explanation.

\begin{tabular}{lrrr}
\hline \hline$\tau$ & \multicolumn{1}{l}{$\mathrm{G} 7$} & $\mathrm{G} 8$ & \multicolumn{1}{l}{$\mathrm{G} 9$} \\
\hline $2^{2}$ & $-7.948(17.8) 10^{-7}$ & $1.458(2.04) 10^{-6}$ & $-8.946(16.7) 10^{-7}$ \\
$2^{3}$ & $-3.115(20.9) 10^{-7}$ & $-6.022(23.9) 10^{-7}$ & $-9.815(19.6) 10^{-7}$ \\
$2^{4}$ & $-4.627(2.64) 10^{-6}$ & $2.891(3.02) 10^{-6}$ & $6.311(24.7) 10^{-7}$ \\
$2^{5}$ & $-6.886(1.60) 10^{-6}$ & $-3.553(39.7) 10^{-7}$ & $-9.777(32.4) 10^{-7}$ \\
$2^{6}$ & $4.847(2.15) 10^{-6}$ & $7.958(4.12) 10^{-6}$ & $6.729(4.26) 10^{-6}$ \\
$2^{7}$ & $-1.192(0.29) 10^{-5}$ & $-1.554(0.58) 10^{-5}$ & $-3.911(5.98) 10^{-6}$ \\
$2^{8}$ & $-2.563(0.41) 10^{-5}$ & $-1.939(0.80) 10^{-5}$ & $-8.900(82.7) 10^{-7}$ \\
$2^{9}$ & $-1.906(0.56) 10^{-5}$ & $3.122(14.1) 10^{-6}$ & $-1.343(1.15) 10^{-5}$ \\
$2^{10}$ & $-9.156(7.90) 10^{-6}$ & $-1.836(19.7) 10^{-6}$ & $-2.701(16.1) 10^{-6}$ \\
$2^{11}$ & $2.095(11.1) 10^{-6}$ & $7.061(27.7) 10^{-6}$ & $2.019(2.26) 10^{-5}$ \\
$2^{12}$ & $-3.466(15.6) 10^{-6}$ & $-7.505(38.9) 10^{-6}$ & $3.240(3.18) 10^{-5}$ \\
$2^{13}$ & $9.711(21.5) 10^{-6}$ & $2.112(3.17) 10^{-5}$ & $-2.428(2.44) 10^{-5}$ \\
$2^{14}$ & $8.670(30.1) 10^{-6}$ & $8.369(44.6) 10^{-6}$ & $-8.337(34.5) 10^{-6}$ \\
$2^{15}$ & $3.692(4.71) 10^{-5}$ & $5.826(6.74) 10^{-5}$ & $-4.166(53.1) 10^{-6}$ \\
$2^{16}$ & $-3.956(6.65) 10^{-5}$ & $1.025(0.95) 10^{-4}$ & $-2.485(7.50) 10^{-5}$ \\
$2^{17}$ & $-1.24(0.94) 910^{-4}$ & $4.591(6.13) 10^{-5}$ & $-5.423(10.6) 10^{-5}$ \\
$2^{18}$ & $-1.782(1.30) 10^{-4}$ & $7.842(8.66) 10^{-5}$ & $8.842(15.0) 10^{-5}$ \\
$2^{19}$ & $-1.579(1.79) 10^{-4}$ & $1.968(1.22) 10^{-4}$ & $-2.139(21.2) 10^{-5}$ \\
$2^{20}$ & $-1.544(2.54) 10^{-4}$ & $1.180(38.0) 10^{-5}$ & $2.337(29.9) 10^{-5}$ \\
$2^{21}$ & $-3.535(3.59) 10^{-4}$ & $-4.704(5.37) 10^{-4}$ & $1.841(4.23) 10^{-4}$ \\
\hline \hline
\end{tabular}

TABLE III. The numerical values of $\mathcal{R}(\tau)$ are tabulated in columns for the generators G7, G8, G9. See Tab. If for an explanation.

\section{APPENDIX B: TIMING RESULTS}

In table $\mathrm{V}$ the typical execution times relative to the generator G1 are given. All generators have been configured to deliver one PRN per function call and no function code has been inlined. Although the figures may scatter between different architectures, compilers and optimization options they should be indicative for the relative performance on work station type computers. It should be mentioned that in the case of combined MLCGs and combined MRGs (G7,G9) a floating point implementation is often much faster than an integer implementation on many modern CPUs. These versions can compete with the fastest generators of table $\mathrm{V}$. 50.

\begin{tabular}{lrr}
\hline \hline$\tau$ & \multicolumn{1}{c}{ G10 } & \multicolumn{1}{c}{ G11 } \\
\hline $2^{2}$ & $-5.345(21.8) 10^{-7}$ & $-1.221(2.46) 10^{-6}$ \\
$2^{3}$ & $1.153(2.56) 10^{-6}$ & $2.644(28.9) 10^{-7}$ \\
$2^{4}$ & $-1.787(3.23) 10^{-6}$ & $6.721(36.5) 10^{-7}$ \\
$2^{5}$ & $-6.273(4.25) 10^{-6}$ & $-4.801(3.49) 10^{-6}$ \\
$2^{6}$ & $1.024(0.53) 10^{-5}$ & $6.012(4.70) 10^{-6}$ \\
$2^{7}$ & $3.864(7.22) 10^{-6}$ & $-8.174(6.43) 10^{-6}$ \\
$2^{8}$ & $-1.085(1.08) 10^{-5}$ & $-1.465(0.89) 10^{-5}$ \\
$2^{9}$ & $-5.065(15.1) 10^{-6}$ & $9.626(17.0) 10^{-6}$ \\
$2^{10}$ & $1.159(2.11) 10^{-5}$ & $5.613(2377) 10^{-8}$ \\
$2^{11}$ & $-6.933(29.6) 10^{-6}$ & $-1.168(3.34) 10^{-5}$ \\
$2^{12}$ & $1.959(4.16) 10^{-5}$ & $-8.471(46.9) 10^{-6}$ \\
$2^{13}$ & $3.068(2.52) 10^{-5}$ & $2.292(3.04) 10^{-5}$ \\
$2^{14}$ & $-1.824(35.6) 10^{-6}$ & $4.688(4.29) 10^{-5}$ \\
$2^{15}$ & $9.589(5.23) 10^{-5}$ & $-4.816(3.40) 10^{-5}$ \\
$2^{16}$ & $7.998(7.39) 10^{-5}$ & $-6.608(4.80) 10^{-5}$ \\
$2^{17}$ & $8.373(10.4) 10^{-5}$ & $-7.858(6.78) 10^{-5}$ \\
$2^{18}$ & $2.910(14.8) 10^{-5}$ & $-2.508(1.83) 10^{-4}$ \\
$2^{19}$ & $-7.407(20.9) 10^{-5}$ & $-7.072(259) 10^{-6}$ \\
$2^{20}$ & $-2.363(29.5) 10^{-5}$ & $2.755(36.7) 10^{-5}$ \\
$2^{21}$ & $9.895(41.7) 10^{-5}$ & $-8.283(5.18) 10^{-4}$ \\
\hline \hline
\end{tabular}

TABLE IV. The numerical values of $\mathcal{R}(\tau)$ are tabulated in columns for the generators G10, G11. See Tab. for an explanation.

\begin{tabular}{lr|lr}
\hline \hline PRNG & rel. time & PRNG & rel. time \\
\hline G1 & $\equiv 1$ & G7 & $\approx 2.2$ \\
G2 & $\approx 1.1$ & G8 & $\approx 0.7$ \\
G3 & $\approx 0.6$ & G9 & $\approx 2.4$ \\
G4 & $\approx 1.4$ & G10 & $\approx 0.7$ \\
G5 & $\approx 0.6$ & G11 & $\approx 0.9$ \\
G6 & $\approx 1.3$ & & \\
\hline \hline
\end{tabular}

TABLE V. Relative execution times of the generators considered in this test.

\section{APPENDIX C: ADDITIONAL RESULTS}

For comparison the performance of the generators G1G11 in the recently proposed n-block test and the random walk test 18 20 has been calculated. For the group of PRNGs which have already been considered in Ref. [18 20 the results were reproduced. The figures for all generators tested newly are reported in Tab. VI. According to Ref. 18 20 the limit of acceptance in the $\chi^{2}$-test has been chosen $\chi^{2}<7.815$ in the case of the random walk test and $\chi^{2}<3.841$ for the $n$-block test. A generator is assumed to pass the test if in at least two of three independent runs the value of $\chi^{2}$ is below the given limit.

The only PRNGs which shows significant deviations from the expected distributions are generators G3 and G5. If the decimation strategy is used then G3 also passes these tests (corresponds to G4).

These results have to be contrasted with the performance of the PRNGs in the $R S$ statistical analysis which is much more stringent in the sense that more generators fail it.

From the presented figures it is obvious that the walk length (block size) in these tests is too small (by orders of magnitude) to catch the severe defects at lags that correspond to the large walk lengths in realistic simulations. 
It is also evident that it is not sufficient to consider only a fixed lag as the amplitude of the deviations can vary strongly with the lag. Finally the $R S$ statistic appears to be superior considering its sensitivity for correlations.

\begin{tabular}{lrrr|rrrr}
\hline \hline PRNG & \multicolumn{2}{c}{$\chi^{2}$ in random walk test } & \multicolumn{3}{c}{$\chi^{2}$ in } & n-block & test \\
\hline G1 & 1.386 & 1.539 & 2.499 & 0.197 & 0.067 & 0.079 \\
G2 & 2.131 & 2.889 & 5.127 & 0.009 & 0.026 & 0.014 \\
G3 & 36.567 & 61.235 & 44.200 & 1.607 & 2.161 & 1.104 \\
G4 & 1.402 & 2.225 & 7.080 & & 0.982 & 0.801 & 1.002 \\
G5 & 433.98 & 490.93 & 424.04 & & 515.46 & 557.06 & 491.57 \\
G6 & 1.883 & 1.958 & 0.780 & 1.797 & 0.152 & 0.214 \\
G7 & 1.764 & 0.329 & 1.093 & 0.397 & 0.488 & 0.002 \\
G8 & 2.378 & 1.289 & 3.497 & 0.160 & 0.764 & 0.024 \\
G9 & 2.275 & 4.663 & 8.249 & 1.592 & 0.008 & 2.598 \\
G10 & 2.634 & 1.699 & 0.979 & 0.325 & 2.550 & 0.341 \\
G11 & 2.368 & 3.858 & 0.239 & 0.452 & 0.035 & 0.817 \\
\hline \hline
\end{tabular}

TABLE VI. Results for three runs of the random walk test (walk length $N=750$ using $10^{6}$ samples) and of the $n$-block test (block size $N=500$ using $3 \times 10^{6}$ samples) 18 20. The framed figures indicate a failure in this test.

\section{ACKNOWLEDGMENTS}

I would like to thank Pierre L'Ecuyer for many valuable discussions and a critical reading of the manuscript. Stimulating talks with Eckhard Pehlke and Ferdinand Evers are also acknowledged.

[1] Software packages for this purpose can be found for instance at NETLIB at http://netlib.att.com/ netlib/random/.

[2] G. Marsaglia, in Computer Science and Statistics: The Interface, Ed.: L. Billard, Elsevier Science Publ., Amsterdam, p. 3, 1985; The software package DIEHARD, $A$ battery of tests of randomness, is vailable via WWW at http://stat.fsu.edu/ geo/diehard.html, 1996; The Marsaglia Random Number CDROM contains 4.8 billion random bits obtained from a combination of several sources.

[3] P. L'Ecuyer, Chap. 4 Random Number Generation in Handbook on Simulation, Ed. Jerry Banks, Wiley, 1997.

[4] D. E. Knuth, The Art of Computer Programming, Volume 2: Seminumerical Algorithms, Addison-Wesley, Reading, MA, 2nd edition, 1981.

[5] P. L'Ecuyer, Ann. Oper. Res. 53, 77, (1994).

[6] G. Marsaglia, Proc. of the Nat. Acad. Sci. 61, 25 (1968).

[7] F. James, Comput. Phys. Commun. 60, 329 (1990).

[8] H. Niederreiter, Random Number Generation and QuasiMonte Carlo Methods, Vol. 63, SIAM, Philadelphia, 1992.

[9] A. M. Ferrenberg, D. P. Landau, and Y. J. Wong, Phys. Rev. Lett. 69, 3382 (1992).
[10] W. Selke, A. L. Talapov, and L. N. Shchur, Pis'ma Zh. Eksp. Teo. Fiz. 58, 684 (1993) [JETP Lett. 58, 665 (193)].

[11] P. Grassberger, J. Phys. A 26, 2796 (1993); Phys. Lett. A 181, 43 (1993).

[12] P. D. Coddington, Int. J. Mod. Phys. C 5, 547 (1994).

[13] F. Schmid, N. B. Wilding, Int. J. Mod. Phys. C 6, 781 (1995).

[14] G. Marsaglia and A. Zaman, Comput. Phys. 8, 117 (1994); Apparently the published C source code for the composite generator mzran13 contains a misprint: the "-" in line 8 should be replaced by a "=".

[15] P. L'Ecuyer, Oper. Res. 44, 816 (1996).

[16] P. L'Ecuyer, Math. Comp. 65, 203 (1996).

[17] M. Matsumoto and Y. Kurita, ACM Trans. Models and Comput. Simul. 2, 179 (1992); ibid., 4, 254 (1994); the 1996 version which improves the lower bit correlations can be obtained from the author.

[18] I. Vattulainen, T. Ala-Nissila, and K. Kankaala, Phys. Rev. Lett. 73, 2513 (1994).

[19] I. Vattulainen, T. Ala-Nissila, and K. Kankaala, Phys. Rev. E 52, 3205 (1995).

[20] I. Vattulainen and T. Ala-Nissila, Comput. Phys. 9, 500 (1995).

[21] I. Vattulainen, K. Kankaala, J. Saarinen, and T. AlaNissila, Comput. Phys. Commun. 86, 209 (1995).

[22] H. E. Hurst, Trans. Am. Soc. Civ. Eng. 116, 770 (1951).

[23] H. E. Hurst, R. Black, Y. M. Sinaika, Long-Term Storage in Reservoirs: An experimental Study, Constable, London, 1965.

[24] B. B. Mandelbrot and J. W. van Ness, SIAM Review 10, 422 (1968).

[25] W. Feller, Ann. Math. Stat. 22, 427 (1951);

[26] B. B. Mandelbrot and J. R. Wallis, Water Resour. Res. 5, 967 (1969).

[27] P. Bratley, B. L. Fox, and L. E. Schrage, A Guide to Simulation, 2nd edition, Springer-Verlag, New York, 1987.

[28] P. L'Ecuyer, Commun. ACM 33, 85 (1990).

[29] S. Tezuka, Uniform Random Numbers: Theory and Practice, Kluwer Academic Publishers, Norwell, Mass. 195.

[30] D. H. Lehmer, in Proc. 2nd Symp. on Large-Scale Digital Calculating Machinery, Harvard Univ. Press, Cambridge, p. 141 (1951).

[31] S. K. Park and K. W. Miller, Comm. ACM 31, 1192 (1988).

[32] P. L'Ecuyer, Commun. ACM 31, 742 (1988).

[33] R. C. Tausworthe, Math. Comp. 19, 201 (1965).

[34] T. G. Lewis, W. H. Payne, J. Assoc. Comput. Mach. 20, 456 (1973).

[35] S. Kirkpatrick and E. Stoll, J. Comput. Phys. 40, 517 (1981).

[36] W. L. Maier, Dr. Dobb's Journal, May, 152 (1991).

[37] R. M. Ziff, Phys. Rev. Lett. 69, 2670 (1992).

[38] G. Marsaglia and A. Zaman, Ann. Appl. Probability 1, 462 (1991).

[39] R. Couture, P. L'Ecuyer, Math. Comp. 62, 798 (1994).

[40] G. Marsaglia, A. Zaman, and W. W. Tsang, Stat. Prob. Lett. 8, 35 (1990).

[41] L. Blum, M. Blum, and M. Schub, SIAM J. Comput. 15, 364 (1986). 
[42] W. H. Press, B. P. Flannery, S. A. Teukolsky, and W. T. Vetterling, Numerical Recipes in $C$, 2nd edition, Cambridge University Press, 1992.

[43] J. Eichenauer and J. Lehn, Statist. Hefte 27, 315 (1986); J. Eichenauer-Hermann, Int. Stat. Rev. 60, 167 (1992); ibid., 63, 247 (1995).

[44] M. Fushimi, Appl. Math. Lett. 2, 135 (1989).

[45] M. D. McLaren and G. Marsaglia, J. Assoc. Comput. Mach. 12, 83 (1965).

[46] M. Lüscher, Comput. Phys. Commun. 79, 100 (1994).

[47] F. James, Comput. Phys. Commun. 79, 111 (1994).

[48] L. E. Schrage, ACM Trans. Math. Soft. 5, 132-138 (1979).

[49] W. H. Press and S. A. Teukolsky, Comput. Phys. 6, 522 (1992).

[50] P. L'Ecuyer, private communication.

[51] Note added in proof: The recently porposed "Mersenne Twister" of the authors of G11 (a variant of the TGFSR with a giant period $2^{19937}-1$ ) has also been found to pass the RS-test. But one should keep in mind that a long period is a necessary, but not a sufficient condition for a reliable generator. 